\title{
Search for new phenomena in mono-X final states using pp collision data collected in Run-2 by the ATLAS experiment at the LHC
}

\author{
Sergio González Fernández, on behalf of the ATLAS collaboration ${ }^{a, *}$ \\ ${ }^{a}$ Institut de Fisica d'Altes Energies (IFAE), \\ Campus UAB, Facultat Ciencies Nord, 08193 Bellaterra, Barcelona, Spain \\ E-mail: sergio.gonzalez.fernandez@cern.ch
}

\begin{abstract}
Cosmological and astrophysical observations point to the existence of an exotic type of matter known as Dark Matter (DM) that accounts for about $84 \%$ of the mass of the Universe. If DM is realized in the form of weakly interacting massive particles (WIMPs) it could be produced at the LHC $p p$ collider. At colliders, mono-X final states are considered golden channels, for which DM particles are produced in association with energetic jets of hadrons, photons, W/Z bosons or top-quarks. Here we present the latest results from the ATLAS collaboration on the mono-jet and the mono-photon searches in $p p$ collisions at $13 \mathrm{TeV}$ using an integrated luminosity of up to $139 \mathrm{fb}^{-1}$. The results are translated into exclusion limits in different models for new physics including DM simplified models, supersymmetry inspired models, models with large extra spatial dimensions, and models for the production of axion-like particles.
\end{abstract}

40th International Conference on High Energy physics - ICHEP2020

July 28 - August 6, 2020

Prague, Czech Republic (virtual meeting)

${ }^{*}$ Speaker 
Different astronomical observations point to the existence of a non-baryonic form of matter denoted Dark Matter (DM). The main effects caused by DM are gravitational but the existence of a Weakly Interacting Massive Particle (WIMP) is often hypothesized since it leads to the correct relic density for non-relativistic matter in the early universe (WIMP miracle). Such particles could be produced in particle colliders and detected indirectly via initial state radiation (ISR) of the incoming partons or from Standard Model (SM) particles produced in association with the DM. These types of signatures are commonly referred to as mono-X signatures. In these proceedings, the results from mono-jet [1] and mono-photon [2] analyses are presented.

In Simplified Models the WIMP Dark Matter candidate $(\chi)$ interacts with the Standard Model through a mediator $\left(Z_{A}\right)$. These models have four free parameters: the masses of the DM and the mediator $\left(m_{\chi}, m_{Z_{A}}\right)$; the coupling between the DM and the mediator $\left(g_{\chi}\right)$ and the coupling between the mediator and the quarks $\left(g_{q}\right)$. Another proposed DM candidate is the axion, which is the pseudo Nambu-Goldstone boson that arises from breaking the global $U(1)$ Peccei-Quinn symmetry in order to solve the strong $\mathrm{CP}$ problem of QCD. The original axion is quite constrained from theory and other SM extensions with a spontaneously broken global symmetry are also considered and probed by searching the associated Goldstone bosons which are referred to as Axion-Like Particles (ALPs). ALPs signatures for mono-jet and mono-photon can be described with an Effective Field Theory adding a linear basis of operators to the Lagrangian that are suppressed by a scale $f_{a}$ and introducing couplings to gluons $\left(c_{\tilde{G}}\right)$ and other fields $\left(c_{\tilde{W}}, c_{\tilde{B}}\right)$.

The mono-jet analysis suffers from an irreducible background from $Z \rightarrow v v$ processes, followed by $\mathrm{W}+$ jets background contributions for which leptons from semi-leptonic decays are not identified in the final state. This analysis has reached a level of precision (2\%) that requires state-of-theart higher-order QCD and electroweak theoretical predictions. The mono-photon analysis is still limited by statistics and the backgrounds arise from $\mathrm{V}+$ photon $(\mathrm{V}=\mathrm{Z}, \mathrm{W})$ processes and events where jets or electrons as misidentified as photons. The latest results from the ATLAS experiment [3] at the LHC in Run 2 on searches for new phenomena in mono-jet and mono-photon final states are presented in Figure 1 and show a good agreement with Standard model predictions. They are translated into exclusion limits for WIMP Simplified Models and ALPs.
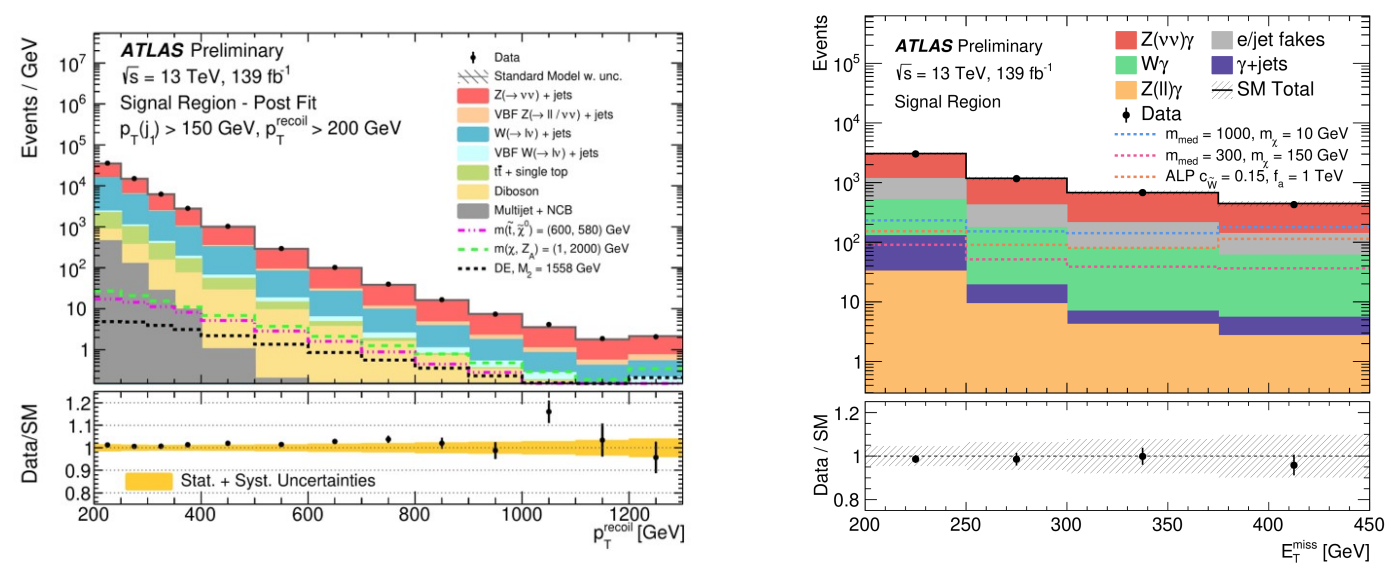

Figure 1: Mono-jet [1] (left) and mono-photon [2] (right) results from the ATLAS experiment in Run 2 compared to the SM predictions. 
As expected from the difference between QCD and electroweak couplings, in the case of the WIMP model, the mono-jet results are more stringent, excluding mediator masses up to $2 \mathrm{TeV}$ for very light WIMPS (Figure 2). The limits in the ALPs model are set on the scale of the effective field theory $\left(f_{a}\right)$ and the coupling of the ALP to gluons $\left(c_{\tilde{G}}\right)$ for the case of mono-jet where values above $8 \times 10^{-6} \mathrm{GeV}^{-1}$ are excluded in the ratio $c_{\tilde{G}} / f_{a}$. In the mono-photon case, a constraint on the coupling of the ALP to two photons is introduced which reduces the number of free parameters and allows the limit to be set on the coupling of the ALP with the weak isospin fields $\left(c_{\tilde{W}}\right)$. In terms of the $c_{\tilde{W}} / f_{a}$ ratio, values above $1.2 \times 10^{-4} \mathrm{GeV}^{-1}$ are excluded. The mono-jet analysis also provides exclusion limits in other models such as SUSY interpretations, Large Extra Dimensions, Dark Energy related models and invisible decays of the Higgs boson.
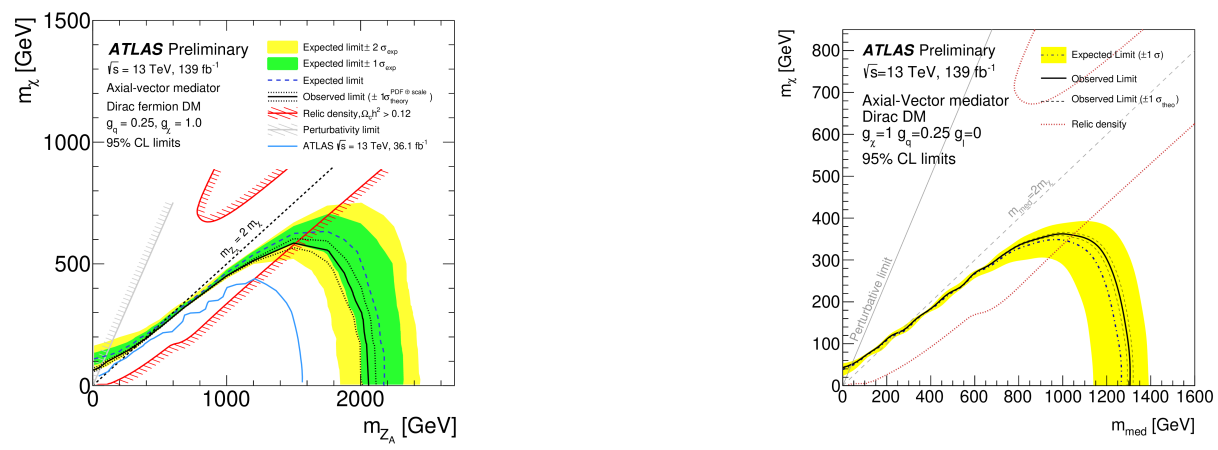

Figure 2: Mono-jet [1] (left) and mono-photon [2] (right) exclusion limits on the WIMP Simplified DM model.
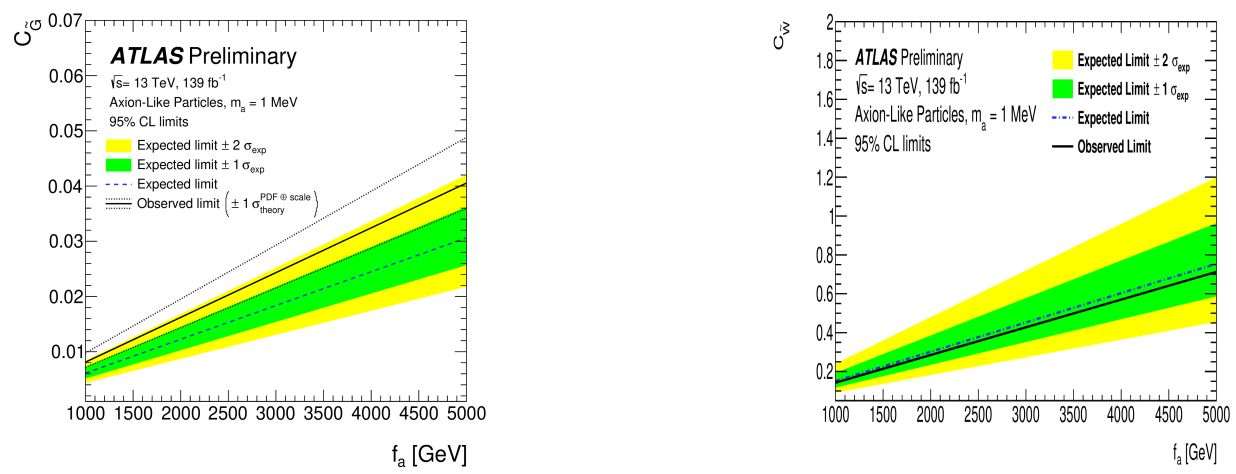

Figure 3: Mono-jet [1] (left) and mono-photon [2] (right) exclusion limits on the ALPs model.

\section{References}

[1] ATLAS Collaboration, Search for new phenomena in events with jets and missing transverse momentum in $p$ collisions at $\sqrt{s}=13 \mathrm{TeV}$ with the ATLAS detector, ATLAS-COM-CONF-2020-059 https://cds.cern.ch/record/2725117.

[2] ATLAS Collaboration, Search for dark matter in association with an energetic photon in pp collisions at $\sqrt{s}=13 \mathrm{TeV}$ with the ATLAS detector, ATLAS-CONF-2020-020 http://cds.cern.ch/record/2720250.

[3] ATLAS Collaboration, The ATLAS experiment at the CERN large hadron collider, Journal of Instrumentation 3 (2008) S08003. 